\title{
CEBPA-CEBPG axis as a novel promising therapeutic target in acute myeloid leukemia
}

\author{
Yin-jun LOU
}

Acta Pharmacologica Sinica (2013) 34: 185-186; doi: 10.1038/aps.2012.191

$\mathrm{D}$ isruption of normal differentiation is an important link in tumorigenesis, but understanding the molecular mechanisms underlying this process is still limited. Acute myeloid leukemia (AML) is characterized by an accumulation of immature myeloid precursor cells in the bone marrow, which has been extensively used as a model for studying genetic/epigenetic regulation of differentiation and cancer progression. Intensive studies using cultured AML cells and animal models have identified wellknown aberrant transcription factors, such as CEBPA, RARa, RUNX1, PU.1, GATA2, etc ${ }^{[1]}$. Consequently, pharmacological restoration of their function represents a promising therapeutic strategy. Noteworthily, all-trans retinoic acid was shown to be capable of inducing the terminal differentiation of immature leukemic promyelocytes, and firstly used clinically in $1988^{[2]}$. Thus, identifying new molecular mechanisms that control myeloid differentiation is fundamental in developing novel anti-leukemic therapies.

In a recent issue of Journal of Clinical Investigation, Meritxell and colleagues have systematically elucidated the role of CEBPA-CEBPG pathway in myeloid differentiation ${ }^{[3]}$. The authors found in the gene expression profile analysis that

Department of Hematology, Institute of Hematology, the First Affiliated Hospital of Zhejiang University, School of Medicine, Hangzhou 310003, China

Correspondence to: Dr Yin-jun LOU

(louyinjun@yahoo.com.cn)
CEBPG mRNA was significantly upregulated in a small subset of AML patients with CEBPA hypermethylation/silencing $(8 / 526)$. They further demonstrated in a CEBPA conditional knockout mice model that ablation of CEBPA led to upregulation of CEBPG in early stem/ progenitor cells. CEBPA could bind to CEBPG proximal promoter and downregulate CEBPG expression by affecting E2F1 transcriptional activity. Furthermore, they showed that CEBPG was downregulated during neutrophilic differentiation in a 32D/G-CSF-R murine cell line model, whereas overexpression of CEBPG blocked granulocytic differentiation. Conversely, inhibition of CEBPG by specific shRNA in vitro or in vivo restored granulocytic differentiation in a CEBPA-knockout early hematopoietic stem/progenitor cells. Intriguingly, the authors found that the DNA demethylating agent 5-aza-2'-deoxycitidine (decitabine) restored the CEBPA-CEBPG balance and induced myeloid differentiation in CEBPA-silenced primary AML blasts. Thus, the authors concluded that targeting the CEBPA-CEBPG pathway represented a novel therapeutic approach in a particular subtype of AML.

CEBPA is important for regulating of myeloid development, directing granulocyte and monocyte differentiation. CEBPA function can be disrupted either through mutation or transcriptional repression. Given the essential role of the CEBPA transcription factor in hematopoiesis, it is crucial to elucidate its mechanisms and the full spectrum of target genes that mediate its activity. This paper demonstrates for the first time that a new CEBPA-CEBPG axis can block myeloid differentiation in subsets of AML, thus revealing novel mechanistic implications for the pathogenesis of AML.

DNA methylation plays a critical role in regulation gene expression during development. However, the mechanisms of how DNA methylation contributes to malignancy development, and how hypomethylation agents exert their effects, are poor understood. This study not only reveals a novel mechanism of AML differentiation failure, but also suggests the necessity of investigating the induction of epigenetic differentiation by DNA hypomethylating agents. DNA hypomethylating agents, including azacitidine and decitabine, have been recently approved for treatment of patients with high-risk myelodysplastic syndrome (MDS) $)^{[4,5]}$. Of particular interest, azacitidine and decitabine, have been used alone or in combination with conventional chemotherapy drugs to treat $\mathrm{AML}^{[6-8]}$. As inhibitors of DNA methylation, the anti-leukemic activity of these agents is believed to reactivate silenced tumor suppressor genes by epigenetic event, but a link between clinical outcomes and the specific gene targets of decitabine is not yet clear. This study provided intriguing data showing the CEBPA-CEBPG axis as a target of decitabine, thus deepened our understanding of the mechanism of the drug's action. 
What' more, the identification of biomarkers of decitabine action would help to select distinct subgroups of AML patients, who may benefit from the drug. At this stage, it will be of great interest to explore the relationship between aberrated CEBPA-CEBPG axis and response to decitabine in future clinical trials.

Recently, cancer genome sequencing studies have revealed recurrent mutation of epigenetic modifying genes, such as DNMT3A, TET2, IDH1/2, and ASXL1 in the pathogenesis of AML ${ }^{[9]}$. Moreover, aberration of epigenetic regulator genes, such as IDH1/2, EZH2, and CREBBP were also found in solid tumors. Increased evidence indicates that carcinogenesis is a multistep process driven by interplay between genomic and epigenomic alterations ${ }^{[10]}$. The direct gene therapy for certain mutatant genes would be especially challenging. Since epigenetic modifications are reversible, they are interesting targets for specific pharmacological interventions. It will be of interest to target downstream and potentially reversible epigenetic consequences with more specific epigenetic agents, such as targeted DNMT inhibitors or specific methylating agents. We are advancing towards an era of personalized medicine in AML, and it is hoped that AML treatment will be greatly improved by integrative pharmacological interventions at both genetic and epigenetic levels in the future, which will provide a useful model for other types of human cancers.

1 Rosenbauer F, Tenen DG. Transcription factors in myeloid development: balancing differentiation with transformation. Nat Rev Immunol 2007; 7 : 105-17.

2 Huang ME, Ye YC, Chen SR, Chai JR, Lu JX, Zhoa L, et al. Use of all-trans retinoic acid in the treatment of acute promyelocytic leukemia. Blood 1988; 72 : 567-72.

3 Alberich-Jorda M, Wouters B, Balastik M, ShapiroKoss C, Zhang H, Diruscio A, et al. C/EBPgamma deregulation results in differentiation arrest in acute myeloid leukemia. J Clin Invest 2012; 122 : 4490-504.

4 Fenaux P, Mufti GJ, Hellstrom-Lindberg E, Santini V, Finelli C, Giagounidis A, et al. Efficacy of azacitidine compared with that of conventional care regimens in the treatment of higher-risk myelodysplastic syndromes: a randomised, open-label, phase III study. Lancet Oncol 2009; 10: 223-32.

5 Kantarjian H, Issa JP, Rosenfeld CS, Bennett JM, Albitar M, DiPersio J, et al. Decitabine improves patient outcomes in myelodysplastic syndromes: results of a phase III randomized study. Cancer 2006; 106: 1794-803.

6 Fenaux P, Mufti GJ, Hellstrom-Lindberg E, Santini V, Gattermann N, Germing U, et al. Azacitidine prolongs overall survival compared with conventional care regimens in elderly patients with low bone marrow blast count acute myeloid leukemia. J Clin Oncol 2010; 28: 562-9.

7 Scandura JM, Roboz GJ, Moh M, Morawa E, Brenet $\mathrm{F}$, Bose JR, et al. Phase 1 study of epigenetic priming with decitabine prior to standard induction chemotherapy for patients with AML. Blood 2011; 118: 1472-80.

8 Blum W, Garzon R, Klisovic RB, Schwind S, Walker $A$, Geyer S, et al. Clinical response and miR29b predictive significance in older AML patients treated with a 10-day schedule of decitabine. Proc Natl Acad Sci U S A 2010; 107: 7473-8.

9 Shih AH, Abdel-Wahab O, Patel JP, Levine RL. The role of mutations in epigenetic regulators in myeloid malignancies. Nat Rev Cancer 2012; 12: 599-612.

10 Turcan S, Rohle D, Goenka A, Walsh LA, Fang F, Yilmaz E, et al. IDH1 mutation is sufficient to establish the glioma hypermethylator phenotype. Nature 2012; 483: 479-83. 